\title{
Metabolic markers in blood can separate prostate cancer from benign prostatic hyperplasia
}

Guro F Giskeødegård ${ }^{1,2}$, Ailin Falkmo Hansen ${ }^{1}$, Helena Bertilsson ${ }^{3,4}$, Susana Villa Gonzalez ${ }^{5}$, Kåre Andre Kristiansen $^{6}$, Per Bruheim ${ }^{6}$, Svein A Mjøs ${ }^{7}$, Anders Angelsen ${ }^{1,2}$, Tone Frost Bathen ${ }^{1}$ and May-Britt Tessem ${ }^{*, 1,2}$

${ }^{1}$ Department of Circulation and Medical Imaging, Norwegian University of Science and Technology (NTNU), Trondheim 7491, Norway; ${ }^{2}$ St Olav University Hospital, Trondheim 7006, Norway; ${ }^{3}$ Department of Cancer Research and Molecular Medicine, Norwegian University of Science and Technology (NTNU), Trondheim 7491, Norway; ${ }^{4}$ Department of Urology, NTNU, Trondheim 7491, Norway; ${ }^{5}$ Department of Chemistry, NTNU, Trondheim 7491, Norway; ${ }^{6}$ Department of Biotechnology, NTNU, Trondheim 7491, Norway and ${ }^{7}$ Department of Chemistry, University of Bergen, Bergen 5020, Norway

Background: An individualised risk-stratified screening for prostate cancer (PCa) would select the patients who will benefit from further investigations as well as therapy. Current detection methods suffer from low sensitivity and specificity, especially for separating PCa from benign prostatic conditions. We have investigated the use of metabolomics analyses of blood samples for separating PCa patients and controls with benign prostatic hyperplasia (BPH).

Methods: Blood plasma and serum samples from 29 PCa patient and 21 controls with BPH were analysed by metabolomics analysis using magnetic resonance spectroscopy, mass spectrometry and gas chromatography. Differences in blood metabolic patterns were examined by multivariate and univariate statistics.

Results: By combining results from different methodological platforms, PCa patients and controls were separated with a sensitivity and specificity of $81.5 \%$ and $75.2 \%$, respectively.

Conclusions: The combined analysis of serum and plasma samples by different metabolomics measurement techniques gave successful discrimination of $\mathrm{PC}$ a and controls, and provided metabolic markers and insight into the processes characteristic of PCa. Our results suggest changes in fatty acid (acylcarnitines), choline (glycerophospholipids) and amino acid metabolism (arginine) as markers for PCa compared with $\mathrm{BPH}$.

Prostate-specific antigen (PSA) screening of healthy men for the diagnosis of early prostate cancer $(\mathrm{PCa})$ is an ongoing controversy because of the risk of overtreatment. Results from the ERSPC study, a randomised trial, showed that PSA screening in healthy men between 50 and 69 years of age, reduces PCa mortality (Schroder et al, 2009) but the downstream consequences of potential side effects from treatment; impotence and incontinence, may cause more harm than benefit for the majority of PCa patients. A risk-stratified screening focusing on men with the highest risk of life-threatening disease would select the patient who will benefit from early diagnosis as well as therapy. Taking into consideration the increasing amount of quinolone-resistant bacteria and the risk of infection after transrectal ultrasound (TRUS) guided biopsies, there is a serious need for a multivariate approach of non-invasive diagnostic markers to pinpoint patients who are eligible to further investigation with more invasive techniques such as TRUS-guided biopsies.

Aberrant metabolism is an emerging hallmark of cancer (Cairns et al, 2011; Hanahan and Weinberg Robert, 2011) and metabolomics is the systematic study of the metabolites expressed in cells, 
tissues and biofluids. Metabolomic analyses of biofluids are minimally invasive and easily accessible for disease monitoring (Zhang et al, 2012), and therefore have strong potential as diagnostic tools. Differences in serum or plasma metabolite concentrations between patients and controls have been detected in several cancers, including colorectal cancer (Nishiumi et al, 2012), pancreatic cancer (Kobayashi et al, 2013) and oral cancer (Tiziani et al, 2009). In PCa, a few studies prove evidence of metabolic differences between PCa patients and healthy controls in plasma (Lokhov et al, 2010), serum samples (Zang et al, 2014) and prostatic secretions (Serkova et al, 2008). Citrate, myo-inositol and the polyamine spermine have previously been suggested as metabolic markers of PCa in prostatic secretions.

The aim of this study was to identify metabolic markers in serum and plasma samples from PCa patients and controls with benign prostatic hyperplasia $(\mathrm{BPH})$ using different suitable methodology platforms, including nuclear magnetic resonance spectroscopy (MRS), mass spectrometry (MS) and gas chromatography (GC) analyses. The use of these complementary methodological platforms allows for quantification of a broad range of metabolites. In contrast to previous studies comparing $\mathrm{PCa}$ patients with healthy volunteers, we have looked for metabolic markers in PCa patients compared with controls with clinical BPH. Thus, this study separates two common conditions giving rise to increased PSA levels (Roehrborn et al, 1999; Bohnen et al, 2007) and can be highly valuable for clinical decision making.

\section{MATERIALS AND METHODS}

Study participants. This was a pilot study including $29 \mathrm{PCa}$ patients and 21 controls. All participants had been referred to the urology department at St Olavs Hospital, Trondheim, because of lower urinary tract symptoms (LUTS) and/or elevated PSA. The cancer patients had been diagnosed with PCa through positive findings in TRUS biopsies, and had not yet started cancer treatment. The control group consisted of men with no proven PCa based on PSA levels $<4 \mathrm{ng} \mathrm{ml}^{-1}$, negative findings on digital rectal palpation, ultrasound-verified prostate volume $\left(\mathrm{cm}^{3}\right)$ and no malignant findings in prostate tissue biopsies when biopsies had been acquired. All men in the control group were diagnosed with LUTS because of BPH. Fasting serum and plasma samples were collected from each participant. The samples were stored at $-80^{\circ} \mathrm{C}$ until analysis. Patient and control characteristics are described in Table 1. Prostate-specific antigen, total high-density lipoprotein (HDL), low-density lipoprotein (LDL), triglycerides and cholesterol concentrations were measured on the same serum sample as analysed by MRS/GC. The Gleason score was histologically determined from TRUS biopsies of the prostate, and were in the range 6-10, where high values represent more aggressive cancers. The study was approved by The Regional Committee for Medical and Health Research Ethics (Norwegian Health Region III) and informed written consent was obtained from all study participants.

Magnetic resonance spectroscopy analysis of serum samples. Thawed serum samples $(100 \mu \mathrm{l})$ were mixed with buffer $(100 \mu \mathrm{l})$ $\left(0.075 \mathrm{~mm} \mathrm{Na}_{2} \mathrm{HPO}_{4}, 5 \mathrm{~mm} \mathrm{NaN}_{3}, 5 \mathrm{~mm}\right.$ TSP, $\mathrm{pH}$ 7.4) and analysed in 3-mm NMR tubes. Magnetic resonance spectroscopy analysis was performed on a Bruker Avance III Ultrashielded Plus $600 \mathrm{MHz}$ spectrometer (Bruker BioSpin GmbH, Rheinstetten, Germany) equipped with a $5 \mathrm{~mm}$ QCI Cryoprobe. Experiments were fully automated using a SampleJet in combination with Icon-NMR on Topspin 3.1 (Bruker BioSpin). 1D ${ }^{1} \mathrm{H}$ Nuclear Overhauser effect spectroscopy (NOESY) and Carr-Purcell-Meiboom-Gill (CPMG) spectra with water presaturation were acquired at $311.4 \mathrm{~K}$. The spectra were Fourier transformed to $128 \mathrm{~K}$ after $0.3 \mathrm{~Hz}$ exponential line broadening. Additional 2D NMR spectra (correlation spectroscopy (COSY), total correlation spectroscopy (TOCSY) and heteronuclear single-quantum coherence spectroscopy (HSQC)) were acquired for selected samples for metabolite identification.

The CMPG spectra were transferred to Matlab R2013b (The Mathworks, Inc., Natick, MA, USA) for further processing. The chemical shifts of the spectra were referenced to the left peak of the alanine doublet at 1.47 p.p.m. Spectral baseline was adjusted by setting the lowest point to zero. No peak alignment was performed because of well overlapping peaks. The spectral region between 0.29 and 8.52 p.p.m., excluding the water peak between 4.31 and 5.15 p.p.m. was chosen for further analyses, and the spectra were normalised to equal total area. Metabolites were assigned using Chenomx NMR suite 7.7 (Chenomx Inc., AB, Edmonton, Canada) and the human metabolome database, in addition to $2 \mathrm{D}$ spectra (COSY, TOCSY and HSQC) for confirming certain assignments. Statistical total correlation spectroscopy (Cloarec et al, 2005) was applied for identification of correlating peaks in the spectrum. Individual metabolite peaks in the normalised 1D CPMG spectra were integrated, and the resulting data represents relative intensities. For metabolites with more than one resonance, either the mean or the resonance in a nonoverlapping region of the spectrum was used.

Lipoprotein subclassification from serum 1D NMR spectra. Lipoprotein subclassification was performed from 1D NOESY MR

Table 1. Characteristics of study participants

\begin{tabular}{|c|c|c|c|}
\hline & Patients ( $n=29)$ (mean, range) & Controls ( $n=21)$ (mean, range) & $P$-value \\
\hline Age (years) & $65.8(58-76)$ & $62.6(52-69)$ & 0.119 \\
\hline BMI $\left(\mathrm{kg} \mathrm{m}^{-2}\right)$ & $26.3(18-34)$ & $26.2(21.0-40.4)$ & 0.630 \\
\hline Prostate volume $(\mathrm{ml})$ & $39.6(19-130)$ & $48.7(21-110)$ & 0.082 \\
\hline PSA at diagnosis $\left(\mathrm{ng} \mathrm{ml}^{-1}\right)$ & $11.6(4.3-50.4)$ & $1.2(0.3-2.6)$ & $8.498 \times 10^{-9 *}$ \\
\hline PSA of sample $\left(\mathrm{ng} \mathrm{ml}^{-1}\right)$ & $14.81(5.8-55.9)$ & $1.51(0.4-4.2)$ & $2.263 \times 10^{-9 *}$ \\
\hline PCA3 (score) & $67.0(2-166)$ & $56.6(6-576)$ & $0.003^{*}$ \\
\hline $\mathrm{HDL}\left(\mathrm{mmoll} \mathrm{I}^{-1}\right)$ & $1.55(0.7-2.23)$ & $1.43(0.7-2.01)$ & 0.345 \\
\hline $\mathrm{LDL}\left(\mathrm{mmoll} \mathrm{I}^{-1}\right)$ & $3.24(1.51-5.53)$ & $3.34(2.42-4.96)$ & 0.477 \\
\hline Triglycerides $\left(\mathrm{mmoll}^{-1}\right)$ & $1.11(0.6-2.82)$ & $1.62(0.46-6.07)$ & $0.011^{*}$ \\
\hline Cholesterol $\left(\mathrm{mmoll}^{-1}\right)$ & $5.28(3.1-7.3)$ & $5.46(4.3-7.1)$ & 0.602 \\
\hline Tumour Gleason score & $7.0(6-9)$ & NA & NA \\
\hline
\end{tabular}


spectra from the serum samples. This method measures the concentration of cholesterol, phospholipids, triglycerides and/or apolipoproteins A1, A2 and B in the serum sample (total values) and in each of the lipoprotein subfractions (total very low-density lipoproteins (VLDL), intermediate density lipoproteins (IDL), LDL and HDL, in addition to the subclasses VLDL-1-6, LDL-1-6 and HDL-1-4). The analysis is based on a partial least squares (PLS) regression model (Petersen et al, 2005; Mihaleva et al, 2014). The analysis was performed by Bruker BioSpin. The internal validity of the measurements was assessed by comparison with the standard laboratory measurements of total serum triglycerides, cholesterol, LDL and HDL cholesterol.

Mass spectrometry analysis of plasma. Targeted analysis of plasma samples was performed on a Acquity UPLC-I Class system coupled to a Xevo TQS mass spectrometer (Waters, Milford, MA, USA) using the AbsoluteIDQ p180 kit (Biocrates Life Sciences AG, Innsbruck, Austria) according to the manufacturer's instructions. The kit provides quantification of up to 188 metabolites including acylcarnitines, amino acids, hexoses, phospholipids, sphingolipids and biogenic amines. Amino acids and biogenic amines are detected and quantified by stable isotopes in LC-MS/MS mode, while the remaining metabolites are detected and semi-quantified in flow injection analysis (FIA) mode. Flow in the LC method was optimised to $0.8 \mathrm{ml} \mathrm{min}^{-1}$. Metabolites detected in LC-MS/MS mode were integrated in TargetLynx (Waters). Results from TargetLynx were together with results from the FIA analysis quantified and validated using the MetIDQ software (Biocrates Life Sciences AG).

Quantification of fatty acids in serum by gas chromatography. Serum samples were derivatised to fatty acid methyl esters (FAME) by direct esterification according to Meier et al (2006). The sample $(200 \mu \mathrm{l})$ was weighed into screw-capped reaction tubes, water was evaporated under nitrogen and internal standard (triheptadecanoin) in isooctane was added. After evaporating the solvent, $0.5 \mathrm{ml}$ of methanolic $\mathrm{HCl}(2.5 \mathrm{M})$ was added to each tube. The tubes were flushed with nitrogen, sealed and incubated at $90^{\circ} \mathrm{C}$ for $2 \mathrm{~h}$. After cooling to room temperature, half of the methanol was evaporated and $0.5 \mathrm{ml}$ water was added. The samples were extracted twice by $1 \mathrm{ml}$ isooctane and the extracts were diluted to $30 \%$ before analysis on GC.

The FAMEs were quantified by GC with flame ionisation detector as described in (Sciotto and Mjøs, 2012), but with minor adjustments of the temperature programme. The FAME reference mixture GLC-461 (Nu-Chek Prep, Elysian, MN, USA) was analysed as every seventh sample. The handling of GC data was performed in Chrombox C (www.chrombox.org) running under Matlab R2013a (Mathworks). Chromatographic areas were corrected by empirical response factors calculated from the GLC-461 mixture. The serum samples were analysed in two replicates, and the average results are presented. The level of each fatty acid is given in percent, where the data are normalised to a total sum of $100 \%$. The fatty acids were identified by analysing a subset of the samples on GC with mass spectrometric detection as described in (Wasta and Mjøs, 2013). The applied fatty acid nomenclature follows the shorthand notation a:b n-c, where ' $a$ ' designates the number of carbons in the fatty acid chain, ' $b$ ' designates the number of double bonds and ' $c$ ' designates the position of the first double bond relative to the methyl end of the carbon chain.

\section{Statistical data analysis}

Multivariate modelling. A principal component analysis (PCA) were performed to visualise the variation in the data set. Principal component analysis could not discriminate cancer patients from controls, thus supervised analyses were performed to extract possible cancer-related information masked by the large metabolic variations in blood. To discriminate cancer patients from controls and to assess correlations to clinical parameters, multivariate analysis was performed on autoscaled quantified data by orthogonalised PLS discriminant analysis (OPLS-DA) and OPLS. Partial least squares is a regression method for the analysis of multivariate data, defining underlying latent variables (LVs) that maximise the covariance between the input variables and the response variable. Partial least squares-discriminant analysis is the extension of PLS. By orthogonalising the resulting model, all relevant discriminatory variance is put into the first LV (LV1), making interpretations of the model easier. Missing data for some variables were replaced by estimated values using a built-in data imputation algorithm. To avoid overfitting, OPLS-DA and OPLS models were validated by 10 -fold random subset cross-validation repeated 10 times. The number of LVs was chosen based on the first minimum in cross-validated classification error and rootmean-square error for OPLS-DA and OPLS, respectively. The importance of each variable in the loadings of the OPLS-DA was evaluated by variable importance in the projection (VIP) scores (Chong and Jun, 2005), positively reflecting the variable's influence on the classification. Permutation testing was performed to assess the significance of the multivariate models $(n=1000$, significance for $P \leqslant 0.05$ ). For classification models using variable-selected data as input, variable selection was included in the permutation loop, choosing the same number of variables as for the original models. Two samples from MS and MRS analysis were excluded from multivariate analysis because of several missing values and very high lipid content, respectively. Multivariate analyses were performed in Matlab using PLS_toolbox 7.8.2 (Eigenvector Research, Inc., Manson, WA, USA). To reduce the number of input variables for multivariate modelling, variable selection was performed by Wilcoxon testing of individual metabolites. Metabolites with Wilcoxon $P$-value $\leqslant 0.05$ from MRS and MS analyses were combined for further analyses.

Univariate statistics. Differences in metabolite levels between patients and controls were assessed by Wilcoxon rank sum testing (Matlab). For variable-selected metabolites from multivariate models, differences in individual metabolite concentrations between PCa patients and controls were examined by receiver operating characteristics (ROC) analyses (SPSS version 21, IBM SPSS Statistics, Armonk, NY, USA). $P$-values were corrected for multiple testing by Benjamini-Hochberg correction, and $q$-values $\leqslant 0.05$ were considered significant. Multiple testing corrections were performed for each platform/analysis method separately.

Pathway analysis. To extract relevant biological information from the data, MetaboAnalyst 3.0 (Xia et al, 2012) was used for metabolic pathway analyses of the metabolomics data from MRS and MS combined. Pathway analysis integrates metabolite set enrichment analysis and pathway topology analysis to extract biologically meaningful information from the data. A table of concentrations for all metabolites was used as input for the analysis, and the data were log transformed and autoscaled before analysis. The pathway analysis was performed through a pathway enrichment analysis using a global test and a pathway topology analysis using a relative betweenness centrality measure.

\section{RESULTS}

\section{Metabolite quantification of serum and plasma samples}

Magnetic resonance spectroscopy of serum. From MRS of serum samples, 28 metabolites were semi-quantified (Supplementary Table 1). Twelve of the metabolites, including amino acids, lipids and metabolites involved in energy metabolism, had significantly 
different levels between patients and controls by testing of individual metabolite levels $(P \leqslant 0.05)$.

Lipoprotein subclassification from MRS spectra. Lipoprotein subclassification resulted in a data set of 105 variables (Supplementary Table 2). The correlations between MRS measured and laboratory measured of triglycerides, cholesterol, LDL and HDL were $r=0.98,0.97,0.95$ and 0.97 , respectively. The levels of 23 variables from lipoprotein subclassification were significantly different between patients and controls $(P \leqslant 0.05)$, including total triglyceride levels and subclasses of total VLDL, VLDL-1-3 (triglycerdies, cholesterol, free cholesterol and phospholipids), VLDL-4 (phospholipids) and VLDL-5 (cholesterol, free cholesterol and phospholipids), in addition to total IDL (free cholesterol and phospholipids) (Supplementary Table 2).

Mass spectrometry analysis of plasma. Mass spectrometry analysis of plasma samples identified 181 metabolites. Of these, 39 metabolites had missing values or values under the limit of detection for $30-100 \%$ of the samples, and were excluded from further analysis, resulting in a list of 142 quantified or semiquantified metabolites (Supplementary Table 3). Fourteen of the MS measured metabolites, including acylcarnitines, amino acids, biogenic amines and glycerophospholipids, had significantly different levels between patients and controls $(P \leqslant 0.05)$.

Gas chromatography analysis of serum. By GC analysis of serum samples, a total of 34 fatty acids were semi-quantified (Supplementary Table 4). This method quantified all free and ester-bound fatty acids, including phospholipids and triglycerides. Two fatty acids (18:2 n-6 and 20:0) had significantly higher levels in PCa patients compared with controls $(P \leqslant 0.05)$.

None of the quantified metabolites/variables in serum or plasma were significantly different between patients and controls after multiple testing corrections $(q>0.05)$, possibly due to a large number of variables compared with samples.

Multivariate classification of PCa patients and controls. Orthogonalized PLS discriminant analysis was used to discriminate the metabolic profiles of PCa patients and controls based on the quantified metabolites/variables from the different measurement methods (Table 2). Plasma metabolites quantified by MS gave significant classification models, thus MS metabolic profiles from plasma samples are significantly different between patients and controls. Serum metabolites quantified by magnetic resonance did also give significant classification models, however with a low sensitivity, showing significant differences in the serum metabolic profiles of patients and controls. Lipoprotein subclasses quantified from the magnetic resonance spectra did also result in significant classification models, thus with a low specificity, showing that $\mathrm{PCa}$ patients and controls have significantly different lipoprotein profiles. Fatty acids quantified by GC analysis did not give significant separation of patients and controls.

The combination of 14 and 12 variable-selected metabolites from MRS and MS, respectively, the two most commonly used platforms for metabolite measurements of biofluids, gave the best classification results. The variable-selected metabolites are listed in Table 3. The OPLS-DA scores and loadings for classification of $\mathrm{PCa}$ and control samples using a combination of variable-selected serum MRS metabolites and plasma MS metabolites are shown in Figure 1. The cancer samples are clustered to the left side of the score plot, with low scores on latent variable 1 (LV1), while the control samples have a high LV1 score. The metabolites decanoylcarnitine (c10), tetradecenoylcarnitine (c14:1), octanoylcarnitine (c8), dimethylsulfone, phenylalanine and lysine are of high importance for classification, with increased levels in PCa patients, while the metabolites phosphatidylcholine diacyl C34:4 and lipid2 have higher levels in the controls.

Receiver operating characteristic statistics of metabolites separating PCa patients and controls. The discriminatory power of the individual metabolites selected for OPLS-DA classification from MS and MRS analyses were examined by ROC analysis. Table 3 shows the area under the curve values of the metabolites. These metabolites were selected based on significant $P$-values from Wilcoxon testing; however, none of the metabolites were significantly different between PCa patients and controls after multiple testing corrections $(q>0.05)$.

Correlations to clinical parameters. Correlations between the metabolic data from different methological platforms and the clinical parameters PCA3 in all samples, and Gleason score of the PCa samples, were examined by OPLS analysis. None of the data sets were significantly correlated to PCA3 or the Gleason score $(P>0.05)$.

MetaboAnalyst results. MetaboAnalyst uses the input metabolites to produce a list of the pathways that are most strongly represented by the metabolites. Only metabolites with a KEGG ID can be included in MetaboAnalyst pathway analysis, thus several of the acylcarnitines were not included, and several of the different phospholipids and sphingolipids have the same KEGG IDs. The pathway analysis suggested a number of significantly altered pathways, with the pathways phenylalanine metabolism, aminoacyl-tRNA biosynthesis, nitrogen metabolism, lysine degradation, pantothenate and CoA biosynthesis and arginine and proline metabolism among the top-listed pathways with three or more metabolite hits (Supplementary Figure 1). The listed pathways were significant before multiple testing corrections, while the results approached significance after multiple testing corrections $(q=0.099)$.

Table 2. OPLS-DA classification results for separating metabolic profiles of prostate cancer patients and controls

\begin{tabular}{|c|c|c|c|c|c|c|}
\hline Analysis method & Variables & $\begin{array}{c}\text { Classification } \\
\text { error (\%) }\end{array}$ & Sensitivity (\%) & Specificity (\%) & LVs & $P$-value \\
\hline MS & All & 28.6 & 73.7 & 69.0 & 2 & $0.004^{a}$ \\
\hline MRS & All & 34.8 & 54.1 & 76.3 & 1 & $0.022^{a}$ \\
\hline Lipoprotein subclassification & All & 35.7 & 70.7 & 57.9 & 3 & $0.027^{a}$ \\
\hline GC of fatty acids & All & 45.0 & 64.8 & 45.2 & 1 & 0.278 \\
\hline MS + MRS combined & All & 32.2 & 75.1 & 60.6 & 1 & $0.007^{a}$ \\
\hline MS + MRS combined & Variable selected & 21.6 & 81.5 & 75.2 & 1 & $0.005^{a}$ \\
\hline
\end{tabular}


Table 3. Variable-selected metabolites used for classification of prostate cancer patients and controls

\begin{tabular}{|c|c|c|c|c|}
\hline Metabolite & Platform & AUC & Direction in cancer & $P$-value \\
\hline Decanoylcarnitine (C10) & MS & 0.73 & $\uparrow$ & $0.006^{*}$ \\
\hline Tetradecenoylcarnitine (C14:1) & MS & 0.70 & $\uparrow \uparrow$ & $0.021^{*}$ \\
\hline Octanoylcarnitine (C8) & MS & 0.73 & $\uparrow$ & $0.022^{\star}$ \\
\hline Nonanoylcarnitine (C9) & MS & 0.72 & $\uparrow$ & $0.009^{*}$ \\
\hline Arg & MS & 0.67 & $\uparrow$ & $0.039 *$ \\
\hline Kynurenine & MS & 0.72 & $\uparrow$ & $0.009^{*}$ \\
\hline lysoPC a C18:0 & MS & 0.68 & $\downarrow$ & $0.039^{*}$ \\
\hline lysoPC a C20:4 & MS & 0.69 & $\downarrow$ & $0.025^{\star}$ \\
\hline PC aa C34:4 & MS & 0.70 & $\downarrow$ & $0.019 *$ \\
\hline PC aa C38:5 & MS & 0.69 & $\downarrow$ & $0.026^{*}$ \\
\hline PC aa C40:4 & MS & 0.67 & $\downarrow$ & $0.045^{\star}$ \\
\hline 2-Methylglutarate & MRS & 0.70 & $\uparrow \uparrow$ & $0.015^{*}$ \\
\hline Lipid2 & MRS & 0.70 & $\downarrow$ & $0.017^{\star}$ \\
\hline $\mathrm{Gln}+\mathrm{Glu}$ & MRS & 0.70 & $\uparrow$ & $0.031^{*}$ \\
\hline Glutamate & MRS & 0.66 & $\uparrow$ & $0.049^{*}$ \\
\hline Pyruvate & MRS & 0.71 & $\uparrow \uparrow$ & $0.015^{\star}$ \\
\hline Lysine & MRS & 0.71 & $\uparrow$ & $0.015^{\star}$ \\
\hline Dimethylsulfone & MRS & 0.74 & $\uparrow$ & $0.006^{*}$ \\
\hline Histidine & MRS & 0.69 & $\uparrow$ & $0.024^{\star}$ \\
\hline Glucose & MRS & 0.68 & $\uparrow$ & $0.039 *$ \\
\hline Tyrosine & MRS & 0.67 & $\uparrow$ & $0.037^{\star}$ \\
\hline
\end{tabular}
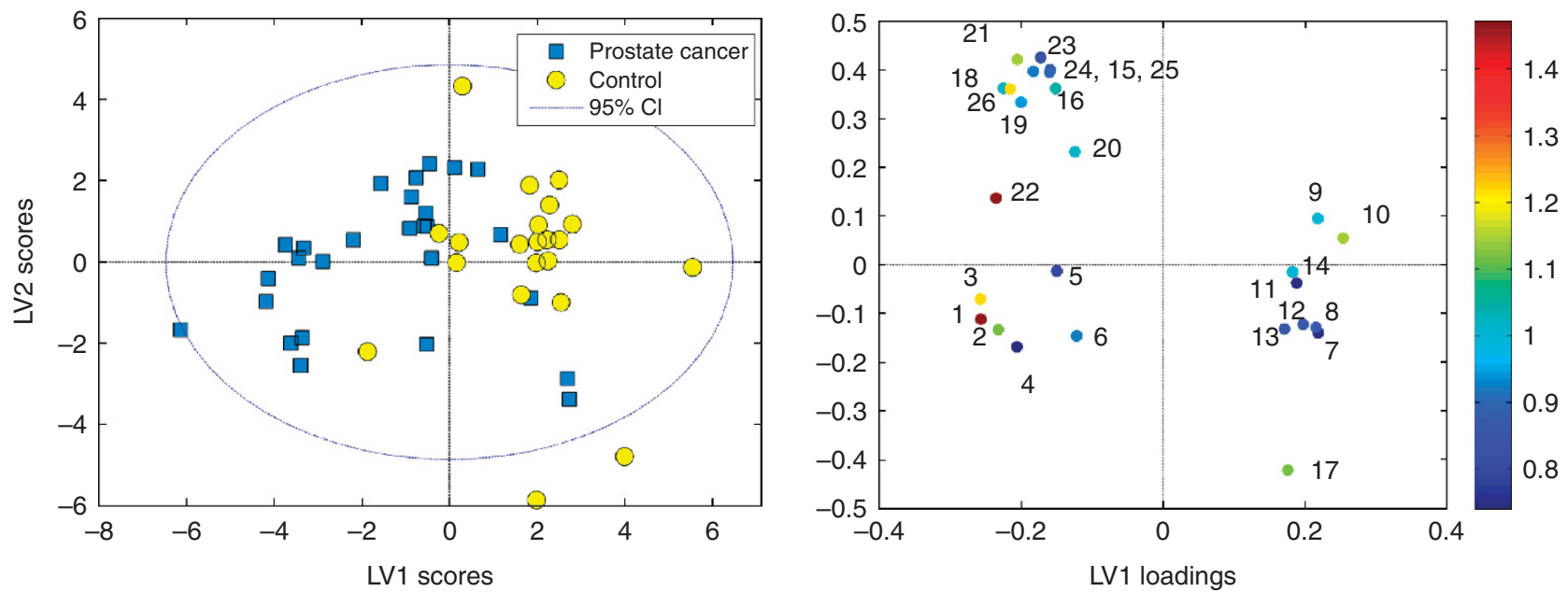

Figure 1. Orthogonalised PLS discriminant analysis scores and loadings plot separating prostate cancer patients and controls based on serum and plasma metabolites from MRS and MS, respectively. The loadings are coloured according to the VIP scores. The metabolites are numbered accordingly: 1: decanoylcarnitine (C10); 2: tetradecenoylcarnitine (C14:1); 3: octanoylcarnitine (C8); 4: nonanoylcarnitine (C9); 5: arginine; 6: kynurenine; 7: lysophosphatidylcholine acyl (lysoPC a) C16:0; 8: lysoPC a C18:0; 9: lysoPC a C20:4; 10: phosphatidylcholine diacyl (PC aa) C34 : 4; 11: PC aa C38:5; 12: PC aa C40:4; 13: PC aa C40:5; 14: phosphatidylcholine acyl-alkyl (PC ae) C38: 2; 15: valine; 16: 2-methylglutarate; 17: lipid2; 18: Gln + Glu; 19: glutamate; 20: pyruvate; 21: lysine; 22: dimethylsulfone; 23: histidine; 24: glucose; 25: tyrosine; and 26: phenylalanine. 


\section{DISCUSSION}

In this study, blood samples were analysed by several methodology platforms, which enabled quantification of different molecular classes of metabolites, including amino acids, fatty acids, biogenic amines and lipoproteins. By combining MRS results from serum and MS results from plasma, we were able to separate PCa patients and $\mathrm{BPH}$ controls with a sensitivity and specificity of $81.5 \%$ and $75.2 \%$, respectively. Control study participants had been referred to the urology department with LUTS due to BPH, thus this study represents a highly relevant clinical setting where PSA levels can be misleading.

Four of the acylcarnitines had increased levels in plasma samples from $\mathrm{PCa}$ patients compared with $\mathrm{BPH}$ controls. Acylcarnitines are involved in fatty acid $\beta$-oxidation, and are formed for transport of fatty acids into the inner mitochondrial membrane by conjugation of long-chain fatty acids to carnitine. Inside the mitochondria the fatty acids are catabolised to generate acetyl-CoA, NADH and $\mathrm{FADH}_{2}$. Zha et al (2005) observed an upregulation of perixosomal, but not mitochondrial, fatty acid $\beta$-oxidation by gene expression profiling of PCa tissue. Peroxisomal fatty acid oxidation is required for initial oxidation of very long-chain and branched-chain fatty acids, which cannot be directly oxidised by the mitochondria. The resulting shorter chained products are transported from the peroxisome to the mitochondria for further oxidation by binding to carnitine. Lokhov et al (2010) have previously described metabolic changes quantified by MS in plasma metabolites of PCa patients compared with controls, and found the acylcarnitine dimethylheptanoyl carnitine to be significantly increased in $\mathrm{PCa}$ patients. This specific acylcarnitine was not quantified in our study; however, the result supports the hypothesis of abnormal plasma acylcarnitine levels in $\mathrm{PCa}$. In contrast to most cancers, increased glucose consumption is not as characteristic for PCa as for other cancers, as evidenced by low fluorodeoxyglucose uptake on PET imaging (Hofer et al, 1999; $\mathrm{Yu}$ et al, 2014). Experiments in three different PCa cell lines showed a higher cellular uptake of fatty acids over glucose, suggesting that fatty acid metabolism is a vital energy source for PCa cells (Liu et al, 2010). Increased levels of acylcarnitines in the blood of PCa patients indicate a higher degree of mitochondrial and/or peroxisomal fatty acid metabolism in PCa.

PCa patients had increased serum levels of glucose compared with BPH controls. Serum levels of glucose have been shown to positively correlate with increased cancer risk (Wulaningsih et al, 2013), possibly as a result of metabolic disorders such as insulin resistance and diabetes. However, a study on a large cohort showed a negative association between serum glucose levels and PCa risk, but an increased PCa risk in men with both high glucose and high triglyceride levels (Van Hemelrijck et al, 2011). In a different study, elevated serum glucose levels at the time of PCa diagnosis was associated with an increased risk of recurrence compared with patients with a normal glucose level (Wright et al, 2013). Further studies are needed to fully map the relationship between serum glucose levels and PCa development and prognosis.

Eight different glycerophospholipids had decreased levels in plasma from PCa patients compared with BPH controls. These include three lysophosphatidylcholines and five phosphatidylcholines. Glycerophospholipids are main constituents of biological membranes. Phosphatidylcholines are phospholipids with a choline head group, while lysophosphatidylcholines are derived from phosphatidylcholines through partial hydrolysis removing one of the fatty acids. Phosphatidylcholines are synthesised through the Kennedy pathway involving the choline containing metabolites free choline, phosphocholine (PCho) and glycerophosphocholine (GPC). Abnormal choline metabolism is frequently observed in cancers (Ackerstaff et al, 2003; Glunde et al, 2011), and we have previously described increased levels of the choline containing metabolites in PCa tissue (Giskeødegård et al, 2013). Decreased plasma levels of phosphatidylcholines and lysophosphatidylcholines might be reflecting the abnormal choline metabolism in PCa tissue. Lokhov et al (2010) did not detect significant differences between PCa patients and controls in any of the quantified phospholipids in their study, which they explain could be due to including early stage cancers and/or lower instrument sensitivity. Decreased levels of lysophosphatidylcholines have however previously been detected in serum samples from PCa patients compared with controls (Osl et al, 2008) and in plasma from colorectal cancer patients (Zhao et al, 2007). Changes in lysophospholipid levels are suggested to result from their activation of specific cell-surface $G$ protein coupled receptors that initiate cell growth, proliferation, and survival pathways (Mills and Moolenaar, 2003; Zhao et al, 2007), properties characteristic of cancer growth.

Eight amino acids were increased in blood samples from PCa patients, and phenylalanine metabolism, lysine degradation and arginine and proline metabolism were suggested as significantly altered pathways by MetaboAnalyst. However, several of these amino acids were only increased in the serum samples by MRS, and not in plasma by MS analysis. This might be because of differences in amino acid concentrations between serum and plasma (Chuang et al, 1998), because of matrix effects or because of differences in sample preparations and quantification methods. Both decreased and increased plasma amino acid levels have been described in cancer patients in previous studies (Lai et al, 2005). The amino acid arginine, which was increased in plasma from PCa patients, is highly versatile and involved in several processes. Arginine and its products are critical for the growth of several cancers by enhancing tumour growth, and arginine depletors have been shown effective as anti-cancer drugs in several cancers including PCa (Kim et al, 2009; Delage et al, 2010; Szlosarek, 2014). Further studies on a larger cohort are necessary to validate the findings of increased blood levels of certain amino acids, including arginine.

In our study, the $\mathrm{BPH}$ controls had significantly higher concentrations of triglycerides compared with $\mathrm{PCa}$ patients. As hypertriglyceridemia has been suggested as a risk factor for PCa (Wuermli et al, 2005), the opposite result would have been expected, and the significantly increased triglyceride levels might be a result of the control group not consisting of healthy men, but men diagnosed with BPH. Additionally, we detected lower levels of lipid signals (lipid2) in PCa patients compared with BPH controls in serum samples, and lipoprotein subclassification showed decreased levels of several of the subfractions of total VLDL and its subclasses VLDL-1-5 in patients compared with BPH controls, in addition to decreased levels of IDL subfractions. In contrast, increased levels of VLDL have been observed in breast cancer patients compared with controls (Franky Dhaval et al, 2008). We did not observe any differences in HDL subfractions in our cohort, although low levels of HDL cholesterol have been suggested as a risk factor and prognostic factor for PCa (Kotani et al, 2013).

The fatty acid data quantified by GC analysis were not significantly different between PCa patients and $\mathrm{BPH}$ controls by multivariate analysis. By MS analysis we showed that several of the acylcarnitines are increased in plasma from PCa patients, while several glycerophospholipids are decreased. The GC method does not tell which lipid groups the various fatty acids are bound in, and it is possible that the summed effect cancel out the differences between fatty acids in different lipid groups.

Although the classification models separating cancer patients and $\mathrm{BPH}$ controls were highly significant, none of the individual metabolites were significantly different between the groups after correcting for multiple testing by the false discovery rate approach. This might be because of a relatively low number of included samples compared with the large number of metabolites quantified 
by the combination of MRS, MS and GS analyses. In addition, our results show the beneficial effect of applying multivariate techniques that do not only look at the metabolites individually, but takes into account interactions between the metabolites. Metabolism is complex, and will be affected by several internal and external factors such as genetics, time-of-day variation and food intake, in addition to the individual's health status. It is unlikely that the levels of only one or a few metabolites will be predictive of cancer, but instead the interactions between several co-varying metabolites may provide a better picture of the disease.

In this study, we chose patients with $\mathrm{BPH}$ as a control group. This is a highly relevant clinical setting as BPH is very common in elderly and middle-aged men. Although not significant, there was a trend of larger prostates in the $\mathrm{BPH}$ control group compared with the patients $(P=0.082)$. It is possible that prostate size might affect the metabolic patterns of the prostate that are visible in the individual's blood stream. However, comparing PCa patients to controls with enlarged prostates is a highly relevant problem to be addressed and a typical setting where PSA measurements are not optimal. The control patients with PSA $<4$ may, however, have a small chance of holding undiagnosed $\mathrm{PCa}$, which can affect the metabolic state towards a more PCa like profile and blur the differences between $\mathrm{PCa}$ and $\mathrm{BPH}$. Another challenge with patients typically referred to the urology clinic is the use of medications for unrelated diseases such as high blood pressure, high cholesterol levels and diabetes. This was also the case for our study, where 34 of the study participants were taking different types of medications. However, none had yet started taking any cancer-related medications. We did not observe any systematic effects of the different types of medications in the metabolic profiles of the study participants, but it is conceivable that some of the medications will affect the metabolic profiles measured in the blood. Thus, it is possible that metabolic differences between PCa patients and $\mathrm{BPH}$ controls would have been more prominent if none of the participants were taking medications.

To conclude, this pilot study shows significant differences in the metabolic profiles of blood samples from $\mathrm{PCa}$ patients and $\mathrm{BPH}$ controls. The combined analysis of serum and plasma samples by different measurement techniques gave successful discrimination of patients and controls with $\mathrm{BPH}$, and provided an insight into the metabolic processes characteristic of $\mathrm{PCa}$, with data suggesting changes in fatty acid metabolism and choline metabolism. Blood samples from a larger study cohort are currently being collected for further validation of the metabolic differences between $\mathrm{PCa}$ patients and controls with clinically relevant $\mathrm{BPH}$.

\section{ACKNOWLEDGEMENTS}

The MRS acquisitions were performed at the MR Core Facility, Norwegian University of Science and Technology. We also acknowledge the Clinical Research Facility, St Olav University Hospital for sample collection and The Regional Biobank of Central Norway, St Olav University Hospital for sample storage. Bruker BioSpin GmbH, Rheinstetten is acknowledged for access to lipoprotein subclass analysis.

\section{CONFLICT OF INTEREST}

The authors declare no conflict of interest.

\section{REFERENCES}

Ackerstaff E, Glunde K, Bhujwalla ZM (2003) Choline phospholipid metabolism: a target in cancer cells? J Cell Biochem 90(3): 525-533.
Bohnen AM, Groeneveld FP, Bosch JL (2007) Serum prostate-specific antigen as a predictor of prostate volume in the community: the Krimpen Study. Eur Urol 51(6): 1645-1653.

Cairns RA, Harris IS, Mak TW (2011) Regulation of cancer cell metabolism. Nat Rev Cancer 11(2): 85-95.

Chong I-G, Jun C-H (2005) Performance of some variable selection methods when multicollinearity is present. Chemometr Intell Lab Syst 78(1-2): 103-112.

Chuang C-K, Lin S-P, Lin Y-T, Huang F-Y (1998) Effects of anticoagulants in amino acid analysis: comparisons of heparin, EDTA, and sodium citrate in vacutainer tubes for plasma preparation. Clin Chem 44(5): 1052-1056.

Cloarec O, Dumas M-E, Craig A, Barton RH, Trygg J, Hudson J, Blancher C, Gauguier D, Lindon JC, Holmes E, Nicholson J (2005) Statistical total correlation spectroscopy: an exploratory approach for latent biomarker identification from metabolic 1H NMR data sets. Anal Chem 77(5): 1282-1289.

Delage B, Fennell DA, Nicholson L, McNeish I, Lemoine NR, Crook T, Szlosarek PW (2010) Arginine deprivation and argininosuccinate synthetase expression in the treatment of cancer. Int J Cancer 126(12): 2762-2772.

Franky Dhaval S, Shilin Nandubhai S, Pankaj Manubhai S, Patel HR, Prabhudas Shankerbhai P (2008) Significance of alterations in plasma lipid profile levels in breast cancer. Integr Cancer Ther 7(1): 33-41.

Giskeødegård GF, Bertilsson H, Selnæs KM, Wright AJ, Bathen TF, Viset T, Halgunset J, Angelsen A, Gribbestad IS, Tessem M-B (2013) Spermine and citrate as metabolic biomarkers for assessing prostate cancer aggressiveness. PLoS One 8(4): e62375.

Glunde K, Bhujwalla ZM, Ronen SM (2011) Choline metabolism in malignant transformation. Nat Rev Cancer 11(12): 835-848.

Hanahan D, Weinberg Robert A (2011) Hallmarks of cancer: the next generation. Cell 144(5): 646-674.

Hofer C, Laubenbacher C, Block T, Breul J, Hartung R, Schwaiger M (1999) Fluorine-18-fluorodeoxyglucose positron emission tomography is useless for the detection of local recurrence after radical prostatectomy. Eur Urol 36(1): 31-35.

Kim RH, Coates JM, Bowles TL, McNerney GP, Sutcliffe J, Jung JU, GandourEdwards R, Chuang FYS, Bold RJ, Kung H-J (2009) Arginine deiminase as a novel therapy for prostate cancer induces autophagy and caspaseindependent apoptosis. Cancer Res 69(2): 700-708.

Kobayashi T, Nishiumi S, Ikeda A, Yoshie T, Sakai A, Matsubara A, Izumi Y, Tsumura H, Tsuda M, Nishisaki H, Hayashi N, Kawano S, Fujiwara Y, Minami H, Takenawa T, Azuma T, Yoshida M (2013) A novel serum metabolomics-based diagnostic approach to pancreatic cancer. Cancer Epidemiol Biomarkers Prev 22(4): 571-579.

Kotani K, Sekine Y, Ishikawa S, Ikpot IZ, Suzuki K, Remaley AT (2013) Highdensity lipoprotein and prostate cancer: an overview. J Epidemiol 23(5): 313-319.

Lai H-S, Lee J-C, Lee P-H, Wang S-T, Chen W-J (2005) Plasma free amino acid profile in cancer patients. Semin Cancer Biol 15(4): 267-276.

Liu Y, Zuckier LS, Ghesani NV (2010) Dominant uptake of fatty acid over glucose by prostate cells: a potential new diagnostic and therapeutic approach. Anticancer Res 30(2): 369-374.

Lokhov P, Dashtiev M, Moshkovskii S, Archakov A (2010) Metabolite profiling of blood plasma of patients with prostate cancer. Metabolomics 6(1): $156-163$.

Meier S, Mjøs SA, Joensen H, Grahl-Nielsen O (2006) Validation of a one-step extraction/methylation method for determination of fatty acids and cholesterol in marine tissues. J Chromatogr A 1104(1-2): 291-298.

Mihaleva VV, van Schalkwijk DB, de Graaf AA, van Duynhoven J, van Dorsten FA, Vervoort J, Smilde A, Westerhuis JA, Jacobs DM (2014) A systematic approach to obtain validated partial least square models for predicting lipoprotein subclasses from serum NMR spectra. Anal Chem 86(1): 543-550.

Mills GB, Moolenaar WH (2003) The emerging role of lysophosphatidic acid in cancer. Nat Rev Cancer 3(8): 582-591.

Nishiumi S, Kobayashi T, Ikeda A, Yoshie T, Kibi M, Izumi Y, Okuno T, Hayashi N, Kawano S, Takenawa T, Azuma T, Yoshida M (2012) A novel serum metabolomics-based diagnostic approach for colorectal cancer. PLoS One 7(7): e40459.

Osl M, Dreiseitl S, Pfeifer B, Weinberger K, Klocker H, Bartsch G, Schafer G, Tilg B, Graber A, Baumgartner C (2008) A new rule-based algorithm for identifying metabolic markers in prostate cancer using tandem mass spectrometry. Bioinformatics 24(24): 2908-2914. 
Petersen M, Dyrby M, Toubro S, Engelsen SB, Norgaard L, Pedersen HT, Dyerberg J (2005) Quantification of lipoprotein subclasses by proton nuclear magnetic resonance-based partial least-squares regression models. Clin Chem 51(8): 1457-1461.

Roehrborn CG, Boyle P, Gould AL, Waldstreicher J (1999) Serum prostatespecific antigen as a predictor of prostate volume in men with benign prostatic hyperplasia. Urology 53(3): 581-589.

Schroder FH, Hugosson J, Roobol MJ, Tammela TL, Ciatto S, Nelen V, Kwiatkowski M, Lujan M, Lilja H, Zappa M, Denis LJ, Recker F, Berenguer A, Maattanen L, Bangma CH, Aus G, Villers A, Rebillard X, van der Kwast T, Blijenberg BG, Moss SM, de Koning HJ, Auvinen A (2009) Screening and prostate-cancer mortality in a randomized European study. N Engl J Med 360(13): 1320-1328.

Sciotto C, Mjøs SA (2012) Trans isomers of EPA and DHA in omega-3 products on the European market. Lipids 47(7): 659-667.

Serkova NJ, Gamito EJ, Jones RH, O’Donnell C, Brown JL, Green S, Sullivan H, Hedlund T, Crawford ED (2008) The metabolites citrate, myo-inositol, and spermine are potential age-independent markers of prostate cancer in human expressed prostatic secretions. Prostate 68(6): 620-628.

Szlosarek PW (2014) Arginine deprivation and autophagic cell death in cancer. Proc Natl Acad Sci USA 111(39): 14015-14016.

Tiziani S, Lopes V, Günther UL (2009) Early stage diagnosis of oral cancer using 1H NMR-based metabolomics. Neoplasia 11(3): 269-276.

Van Hemelrijck M, Garmo H, Holmberg L, Walldius G, Jungner I, Hammar N, Lambe M (2011) Prostate cancer risk in the Swedish AMORIS study: the interplay among triglycerides, total cholesterol, and glucose. Cancer 117(10): 2086-2095.

Wasta Z, Mjøs SA (2013) A database of chromatographic properties and mass spectra of fatty acid methyl esters from omega-3 products. J Chromatogr A 1299(0): 94-102.

Wright JL, Plymate SR, Porter MP, Gore JL, Lin DW, Hu E, Zeliadt SB (2013) Hyperglycemia and prostate cancer recurrence in men treated for localized prostate cancer. Prostate Cancer Prostatic Dis 16(2): 204-208.

Wuermli L, Joerger M, Henz S, Schmid HP, Riesen WF, Thomas G, Krek W, Cerny T, Gillessen S (2005) Hypertriglyceridemia as a possible risk factor for prostate cancer. Prostate Cancer Prostatic Dis 8(4): 316-320.

Wulaningsih W, Holmberg L, Garmo H, Zethelius B, Wigertz A, Carroll P, Lambe M, Hammar N, Walldius G, Jungner I, Van Hemelrijck M (2013) Serum glucose and fructosamine in relation to risk of cancer. PLoS One 8(1): e54944.

Xia J, Mandal R, Sinelnikov, Broadhurst D, Wishart DS (2012) MetaboAnalyst 2.0-a comprehensive server for metabolomic data analysis. Nucleic Acids Res 40(Web Server issue): W127-W133.

Yu CY, Desai B, Ji L, Groshen S, Jadvar H (2014) Comparative performance of PET tracers in biochemical recurrence of prostate cancer: a critical analysis of literature. Am J Nuclear Med Mol Imaging 4(6): 580-601.

Zang X, Jones CM, Long TQ, Monge ME, Zhou M, Walker LD, Mezencev R, Gray A, McDonald JF, Fernández FM (2014) Feasibility of detecting prostate cancer by ultraperformance liquid chromatography-mass spectrometry serum metabolomics. J Proteome Res 13(7): 3444-3454.

Zha S, Ferdinandusse S, Hicks JL, Denis S, Dunn TA, Wanders RJ, Luo J, De Marzo AM, Isaacs WB (2005) Peroxisomal branched chain fatty acid $\beta$-oxidation pathway is upregulated in prostate cancer. Prostate 63(4): 316-323.

Zhang A, Sun H, Wang X (2012) Serum metabolomics as a novel diagnostic approach for disease: a systematic review. Anal Bioanal Chem 404(4): 1239-1245.

Zhao Z, Xiao Y, Elson P, Tan H, Plummer SJ, Berk M, Aung PP, Lavery IC, Achkar JP, Li L, Casey G, Xu Y (2007) Plasma lysophosphatidylcholine levels: potential biomarkers for colorectal cancer. J Clin Oncol 25(19): 2696-2701.

This work is published under the standard license to publish agreement. After 12 months the work will become freely available and the license terms will switch to a Creative Commons AttributionNonCommercial-Share Alike 4.0 Unported License.

Supplementary Information accompanies this paper on British Journal of Cancer website (http://www.nature.com/bjc) 